\title{
Autonomic control of blood circulation in patients undergoing elective carotid endarterectomy
}

\author{
Karel Vykoupila, Jan Galuszka ${ }^{a}$, Petr Drac ${ }^{b}$, Milos Taborsky ${ }^{a}$
}

Background. Internal carotid artery stenosis (ICAS) is associated with significantly higher risk of stroke. Autonomic
function can be impaired in the presence of atheroma in the carotid sinus region. Two parameters of autonomic ner-
vous system (ANS) function e.g. heart rate variability (HRV) and baroreflex sensitivity (BRS) are respected predictors of
cardiovascular prognosis. We assessed the effect of elective unilateral carotid endarterectomy (CEA) on cardiovascular
autonomic functions as a major prognostic factor for cardiovascular health. Methods. Nineteen patients indicated for CEA underwent formal autonomic assessment in the laboratory. Hemodynamic profiles, HRV and BRS were evaluated with the dedicated high-tech device Task Force Monitor before surgery (day-1) and postoperatively (day $3 \pm 1$ ). Data were obtained during 5 min orthostatic challenge and subsequent 5 min in a supine position.

Results. There were no significant early postoperative changes in evaluated parameters after CEA. There was a mild decrease of blood pressure and therefore only a slight increase in BRS. It was also possible to observe a rise in the value of total power and high frequency power.

Conclusion. In the early postoperative period, healing processes are occurring and the sympatho-vagal interaction is probably still unbalanced. Given the considerable clinical potential of BRS and HRV measurement, further short-term and, more importantly, long-term investigations are needed.

Key words: internal carotid artery stenosis, autonomic functions, Task Force Monitor, baroreflex sensitivity, heart rate variability

Received: May 13, 2017; Accepted with revision: October 17, 2017; Available online: October 27, 2017 https://doi.org/10.5507/bp.2017.045

${ }^{a}$ Department of Internal Medicine I - Cardiology, University Hospital Olomouc, Czech Republic

${ }^{b}$ Department of Surgery II - Vascular and Transplantation Surgery, University Hospital Olomouc, Czech Republic Corresponding author:Karel Vykoupil, e-mail: vykoupilkarel@gmail.com

\section{INTRODUCTION}

Autonomic nervous system function (ANS) dysfunction is significant and often neglected aspect of a wide range of pathological conditions of both the peripheral and central nervous system and many other illnesses.

The arterial baroreflex constantly influences the whole body. The brain stem receives burst-like information of every blood pressure (BP) pulsation from afferent glossopharyngeal and vagal pathways that are sensed by aortic and carotid baroreceptors. Upon each BP pulsation, the efferent parasympathetic pathways of the reflex transport burst-like patterns and the sympathetic part of the reflex sends inhibitory responses ${ }^{1}$.

The heart is continuously affected by both parasympathetic and sympathetic nervous systems. The baroreflex sends the reciprocal responses of the ANS. When BP increases, the afferent baroreflex signal intensifies causing the efferent sympathetic signal to decrease and the efferent parasympathetic signal to increase. When BP lowers, it is the other way around. The baroreflex also constantly adjusts vascular resistance in a similar way: modulating the sympathetic responses in the whole body ${ }^{2}$.

This buffering mechanism is one, and maybe the most relevant function of the baroreflex. The other function is the management of the input of sympathetic and parasympathetic systems on the heart muscle and its conduction system, thus protecting it from stressful events, e.g. arrhythmias. Both functions are critical ${ }^{3}$.

Nowadays BRS is assessed noninvasively by obtaining the heart rate variability (HRV) and BP variability (BPV) from the continuous finger arterial pressure measured by the Peňáz' principle. Because of the respiration and due to the Mayer waves, there is always spontaneous BPV (ref. ${ }^{4}$ ).

The spectral analysis of the heart rate variability (SAHRV) is considered to be a reliable method reflecting not only parasympathetic but also sympathetic activity and the sympatho-vagal balance ${ }^{5,6}$.

The most frequently used parameters of SAHRV are low frequency power (LF power), high frequency power (HF power), the ratio (LF/HF) and the total power (TP). HRV can be measured in a steady state [during spontaneous breathing or during standardized methods with known influence on ANS activity e.g. Valsalva maneuver, deep breathing, orthostatic test, or ortho-clinostatic test (supine-vertical-supine position)] (ref., ${ }^{5,6}$ ).

HRV is affected by many pathological, pharmacological and physiological variables and conditions.

Depressed HRV is a powerful predictor of mortality and of arrhythmic complications (for example, symptom- 
atic sustained ventricular tachycardia) in patients after acute myocardial infarction ${ }^{7,8}$.

Diminished BRS impedes adequate modulation of baroreceptor activity, resulting in increased BPV. Consequently, low BRS has been associated with less favorable prognosis and long-term mortality after myocardial infarction and ischemic stroke $\mathrm{e}^{9-11}$.

ICAS is associated with a significantly higher risk of ipsilateral stroke ${ }^{12}$.

Development of the disease is accelerated by cardiovascular risk factors such as ageing, smoking, hypercholesterolemia, obesity, diabetes mellitus, hypertension and family history of atherosclerosis. The presence of atheroma in the carotid sinus region is associated with a decreased baroreceptor sensitivity involving an impairment of the afferent baroreceptor activity ${ }^{13}$. Carotid endarterectomy (CEA) is considered to be superior to the medical treatment for stroke prevention in symptomatic patients with significant or moderate internal carotid artery stenosis. In asymptomatic patients CEA can be indicated in selected groups of patients with significant internal carotid artery stenosis ${ }^{14}$. The surgery takes place in the immediate vicinity of baroreceptors located in the carotid sinus ${ }^{15}$. It has been suggested that this invasive procedure may affect the function of the baroreceptor. The low BRS might be a result of an impaired arterial extensibility in the affected area ${ }^{16}$. Some studies show that improved BRS may influence the clinical prognosis ${ }^{17}$. Consequently, the removing of atheroma by CEA could improve the carotid baroreflex sensitivity by enabling better stimulation of the receptors and BRS may be clinically useful for assessing the efficacy of this procedure.

We assessed the effect of elective unilateral CEA on cardiovascular autonomic functions as one of the major prognostic factors of cardiovascular health.

\section{METHODS}

Patients $(n=19)$ underwent formal autonomic assessment in the laboratory. All measurements were performed with a dedicated high-tech device Task Force Monitor (TFM). The main area of TFM application is as an automated and computed beat-to-beat analysis of heart rate (HR) [electrocardiogram (ECG)] oscillometric and non-invasive continuous blood pressure measurements (oscBP, contBP). On the basis of these biological signal sources, hemodynamic and autonomic parameters were calculated. The TFM facilitates continuous (beat-to-beat), precise measurements of all parameters ${ }^{18-20}$.

Basic descriptive statistics were used to summarise the data (mean, median, minimum, maximum and SD) and calculated automatically for defined periods.

The clinical characteristics of participants are shown in Table 1.

In all of the participants with either symptomatic or asymptomatic ICAS indicated for elective CEA, HRV, BRS and hemodynamic profiles were evaluated before surgery (day -1 ) and postoperatively (day $3 \pm 1$ ). The data were analyzed from a record during $5 \mathrm{~min}$ of orthostatic
Table 1. Clinical charasteristics of patients.

\begin{tabular}{lc}
\hline Number of patients & 19 \\
Age (mean) & 69.9 years \\
Male/Female (\%) & $63 / 37$ \\
Operated side left/right (\%) & $57 / 43$ \\
Asymptomatic contralateral (\%) ICAS >70\% & 26 \\
Asymptomatic contralateral (\%) ICAS 50-70\% & 26 \\
Symptomatic/asymptomatic ICAS(\%) & $64 / 36$ \\
History of smoking/Nonsmoker (\%) & $57 / 43$ \\
Arterial hypertension (\%) & 78 \\
Diabetes mellitus (\%) & 43 \\
Ischaemic heart disease (\%) & 31 \\
Peripheral artery disease (\%) & 16 \\
Dyslipidemia (\%) & 78 \\
Atrial fibrilation (\%) & 11 \\
Stroke or transient ischaemic attack (\%) & 31 \\
Use of ACE inhibitors (\%) & 68 \\
Use of Beta blockers (\%) & 23 \\
Use of Calcium channel blockers (\%) & 24 \\
\hline
\end{tabular}

challenge and subsequent 5 min of supine position. High frequency (HFnu-RRI), low frequency (LFnu-RRI), average systolic ( $\mathrm{sBP})$, average diastolic ( $\mathrm{dBP})$, average middle ( $\mathrm{mBP}$ ) blood pressure, HR, TP was derived from supine position. BRS was derived from standing position. Variables before and after surgery were compared using $t$-test in case of normal distribution, respectively Wilcoxon test in case of abnormal distribution (parameters, TP, BRS). For testing of normality the Shapiro-Wilk test was used. These tests were performed at the 0.05 level of significance.

\section{RESULTS}

Autonomic functions and their changes pre- and postoperatively are shown in Table 2.

There were no statistically significant differences in evaluated variables after CEA. Low value of total spectral power as well as low value of LF reflecting sympathetic activity before and after surgery. The rising value of TP after CEA is hand in hand with increasing HF component, reflecting vagal activity. Slight rising value of BRS is associated with mild drop in blood pressure.

\section{DISCUSSION}

The many studies evaluating the effect of CEA on baroreflex function have provided wide variety of results, ranging from improved function to no effect or even to its impairment.

Several studies report that baroreceptor function of some patients deteriorates after CEA (ref. ${ }^{21,22}$ ).

Some authors explain the deterioration of baroreceptor function as damage caused to the carotid sinus nerve during the dissection of the artery ${ }^{23}$.

Nouraei assessed the impact of CEA on BP homeo- 
Table 2. Autonomic nervous system parameters.

\begin{tabular}{lcccc}
\hline & Before surgery & After surgery & Change & $P$ \\
\hline LFnu-RRI supine $(54 \pm 4 \%)$ & 45.2 & 39.7 & -5.5 & 0.154 \\
HFnu-RRI supine $(29 \pm 3 \%)$ & 54.7 & 60.1 & 5.4 & 0.158 \\
TP supine $\left(3466 \pm 1018 \mathrm{~ms}^{2}\right)$ & 554 & 1030 & 476 & 0.687 \\
BRS standing $(\mathrm{ms} / \mathrm{mm} \mathrm{Hg})$ & 7.53 & 8.11 & 0.58 & 0.904 \\
Heart frequency $(\mathrm{bpm})$ & 72.5 & 72.8 & 0.3 & 0.896 \\
sBP $(\mathrm{mmHg})$ & 115.6 & 113 & -2.6 & 0.500 \\
dBP $(\mathrm{mmHg})$ & 65.3 & 63.3 & -1.9 & 0.634 \\
$\mathrm{mBP}(\mathrm{mmHg})$ & 84.7 & 82.9 & -1.8 & 0.616 \\
\hline
\end{tabular}

Data are mean values.

Legend: RRI - R-R interval, LFnu - low frequency in normalized units, HFnu - high frequency in normalized units, TP - total spectral power, BRS - baroreflex sensitivity, sBP - average systolic blood pressure, dBP - average diastolic blood pressure, mBP - average middle blood pressure, bpm - beats per min.

stasis and baroreflex function with particular reference to the presence or absence of significant contralateral carotid artery disease in prospective study consisting of 80 patients with symptomatic extra-cranial carotid disease undergoing CEA. According to their findings the CEA impairs BP homeostasis through surgical destruction of the ipsilateral carotid baroreflex mechanism. Patients with contralateral ICAS have a reduced baroreflex reserve and show greater baroreflex dysfunction and hemodynamic instability after CEA (ref. ${ }^{24}$ ). In our study there were $52 \%$ patients with at least $50 \%$ contralateral ICAS.

No difference in a baroreceptor function was observed in a randomized trial where the carotid sinus nerve was either purposely cut or preserved ${ }^{25}$.

Yakhou measured HRV, and variability of systolic arterial pressure, BRS by intra-arterial pressure in 10 patients after CEA and 10 patients after carotid artery stenting (CAS) (follow up for $24 \mathrm{~h}$ ). BRS and parasympathetic activity increased while systolic BP decreased after endovascular treatment. No significant changes were seen in any parameters for CEA $\left(\right.$ ref. $\left.^{26}\right)$.

In another study, the ratio between HF and LF bands as an index of sympatho-vagal balance was assessed in 10 patients after CEA and 12 after CAS (follow up for 4 days). The authors found a relative increase in a sympathetic activity after CEA and a parasympathetic modulation after CAS (ref. ${ }^{27}$ ).

The transient changes in BRS and cerebral autoregulation may be caused by prothrombotic and inflammatory responses after surgery ${ }^{28}$, so the timing of BRS assessment is also important and may be even critical because of the hemodynamic instability observed in the first $6 \mathrm{~h}$ after the surgery ${ }^{29}$.

Long-term follow up is not affected by acute humoral changes after CEA and, therefore, may better show the hemodynamic changes of successful CEA. One long-term observation study showed that improved BRS after surgery led to a reduction in the cardiovascular endpoints ${ }^{30}$.

In the early postoperative period when healing is taking place, the sympatho-vagal interaction is unbalanced. The power to detect significant differences in our study is undoubtedly limited by small sample size and also short follow-up period. The methods used to measure autonom- ic parameters, especially BRS, are often different, thus comparison of findings across the studies are not valid. Another explanation of the discrepancy in findings in the studies might be the different follow-up periods. The BP of all the patients was effectively controlled and therefore there was only limited space for its improvement.

\section{CONCLUSION}

There were no statistically significant differences in evaluated variables after CEA. No hemodynamic instability was observed during the perioperative period. In our opinion, CEA can be considered as a safe method. Given this considerable clinical potential, BRS and HRV measurement methodology and further short-term and, more importantly, long-term investigations are needed to assess this problematic.

Acknowledgement: We would like to thank Iva Buriánková and Pavla Doupalová for their assistance in the autonomic assessment of the patients in the laboratory. Jana Zapletalová for data analysis and interpretation. And last but not least we would like to thank Petr Utíkal and Petr Bachleda for cooperating with us in the recruitment of patients.

Authors contributions: KV, JG, PD reviewed the literature, drafted the manuscript, and contributed to its revision. PD: performed some of the surgeries. MT: enabled us to use the laboratory and the necessary equipment for this study and helped with the patient management.

Conflict of interest statement: The authors state that there are no conflicts of interest regarding the publication of this article.

\section{REFERENCES}

1. Charkoudian N, Rabbitts JA. Sympathetic neural mechanisms in human cardiovascular health and disease. Mayo Clin Proc 2009;84:82230.

2. Benarroch EE. The arterial baroreflex: functional organization and involvement in neurologic disease. Neurology 2008;71:1733-8.

3. Charkoudian N, Dutoit AP, Hart CE, Wallin GB, Curry TB, Joyner 
MJ. Cardiac baroreflex sensitivity is not correlated to sympathetic baroreflex sensitivity within healthy, young humans. Hypertension 2010;56:1118-23.

4. VanDeVooren H, Gademan MG, Swenne CA, TenVoord BJ, Schalij MJ, Van der Wall EE. Baroreflex sensitivity, blood pressure buffering and resonance: what are the links? Computer simulation of healthy subjects and heart failure patients. J Appl Physiol 2007;102:1348-56.

5. Opavský J. Autonomní nervový systém a diabetická autonomní neuropatie. Praha: Galén 2002.

6. Task Force of the European Society of Cardiology and the North American Society of Pacing and Electrophysiology. Heart rate variability: standards of measurement, physiological interpretation, and clinical use. Circulation 1996;93(5):1043-65.

7. Odemuyiwa O, Malik M, Farrell T, Bashir Y, Poloniecki J, Camm J. Comparison of the predictive characteristics of heart rate variability index and left ventricular ejection fraction for all-cause mortality, arrhythmic events and sudden death after acute myocardial infarction. Am J Cardiol 1991;68:434-9.

8. Kleiger RE, Miller JP, Bigger JT, Moss AJ, and the Multicenter PostInfarction Research Group. Decreased heart rate variability and its association with increased mortality after acute myocardial infarction. Am J Cardiol 1987;59:256-62.

9. La Rovere MT, Pinna GD, Hohnloser SH, Marcus FI, Mortara A Nohara R, Bigger JT Jr, Camm AJ, Schwartz PJ, Atrami investigators. Baroreflex sensitivity and heart rate variability in the identification of patients at risk for life-threatening arrhythmias: Implications for clinical trials. Circulation 2001;103:2072-7.

10. Robinson TG, Dawson SL, Eames PJ, Panerai RB, Potter JF. Cardiac baroreceptor sensitivity predicts long-term outcome after acute ischemic stroke. Stroke 2003;34:705-12.

11. La Rovere MT, Maestri R, Robbi E,Caporotondi A, Guazzotti G, Febo $\mathrm{O}$, Pinna GD. Comparison of the prognostic values of invasive and noninvasive assessments of baroreflex sensitivity in heart failure. $J$ Hypertens 2011;29:1546-52.

12. Chen X, Hassan MO, Jones JV, Sleight P, Floras JS. Baroreflex sensitivity and the blood pressure response to beta-blockade. J Hum Hypertens 1999;13:185-90.

13. Akinola A, Mathias CJ, Mansfield A, Thomas D, Wolfe J, Nicolaides AN, Tegos T. Cardiovascular autonomic and plasma catecholamine responses in unilateral and bilateral carotid artery stenosis. J Neuro Neurosurg Psychiatry 1999;67:428-32.

14. Brott TG, Halperin JL, Abbara S, Bacharach JM, Barr JD, Bush RL, Cates CU, Creager MA, Fowler SB, Friday G, Hertzberg VS, Mclff EB, Moore WS, Panagos PD, Riles TS, Rosenwasser RH, Taylor AJ. 2011 ASA/ACCF/AHA/AANN/AANS/ACR/ASNR/CNS/SAIP/SCAI/SIR/SNIS/ SVM/SVS guideline on the management of patients with extracranial carotid and vertebral artery disease: a report of the American College of Cardiology Foundation/American Heart Association Task Force on Practice Guidelines, and the American Stroke Association, American Association of Neuroscience Nurses, American Association of Neurological Surgeons, American College of Radiology, American Society of Neuroradiology, Congress of Neurological Surgeons, Society of Atherosclerosis Imaging and Prevention, Society for Cardiovascular Angiography and Interventions, Society of Interventional Radiology, Society of Neurolnterventional Surgery,
Society for Vascular Medicine, and Society for Vascular Surgery. J Am Coll Cardiol 2011;57(8):e16-94. doi: 10.1016/j.jacc.2010.11.006

15. Karemaker JM, Wesseling KH. Variability in cardiovascular control: The baroreflex reconsidered. Cardiovasc Eng 2008;8:23-9.

16. Nasr N, Pavy-Le Traon A, Larrue V. Baroreflex sensitivity is impaired in bilateral carotid atherosclerosis. Stroke 2005;36:1891-5.

17. La Rovere, MT, Bigger JT, Marcus Fl, Mortara A, Schwartz PJ. Baroreflex sensitivity and heart-rate variability in prediction of total cardiac mortality after myocardial infarction. ATRAMI (Autonomic Tone and Reflexes After Myocardial Infarction) investigators. Lancet 1998;351:478-84.

18. Fortin J, Habenbacher W, Gruellenberger R, Wach P, Skrabal F. Real time monitor for hemodynamic beat-to-beat parameters and power spectra analysis of biosignals Proceedings of the 20th annual International Conference of the IEEE Engineering in Medicine and Biology Society, Hong Kong, 1998.

19. Fortin J, Klinger Th, Wagner Ch, Sterner $\mathrm{H}$, Madritsch Ch Grüllenberger R, Hacker A, Habenbacher W, Skrabal F. The Task Force Monitor a non-invasive beat-to-beat monitor for hemodynamic and autonomic function of the human bodyProceedings of the 20th annual International Conference of the IEEE Engineering in Medicine and Biology Society, Hong Kong, 1998.

20. Fortin J, Marte W, Grillenberger R. Continuous non-invasive blood pressure monitoring using concetrically interlocking control loops, Comput Biol Med 2006;36:941-57.

21. Dodds SR, Finch D, Chant ADB. Early effect of carotid endarterectomy on arterial blood pressure measured with an ambulatory monitor. Br J Surg 1997;84:1104-6.

22. Angell-James JE. Arterial baroreceptor activity in rabbits with experimental atherosclerosis. Circ Res 1974;34:27-39.

23. Cafferata HT, Merchant RF, DePalma RG. Avoidance of postcarotid endarterectomy hypertension. Ann Surg 1982;196:465-72.

24. Nouraei SA, Al-Rawi PG, Sigaudo-Roussel D, Giussani DA, Gaunt ME. Carotid endarterectomy impairs blood pressure homeostasis by reducing the physiologic baroreflex reserve. J Vasc Surg 2005;41:631-7.

25. Towne JB, Bernhard VM. The relationship of postoperative hypertension to complications following carotid endarterectomy. Surgery 1980;88:575-80.

26. Yakhou L, Constant I, Merle JC, Laude D, Becquemin JP, Duvaldestin P. Noninvasive investigation of autonomic activity after carotid stenting or carotid endarterectomy. J Vasc Surg 2006;44(3):472-9.

27. Demirci M, Saribas O, Uluç K, Ay H. Carotid artery stenting and endarterectomy have different effects on heart rate variability. J Neurol Sci 2006;241(1-2):45-51.

28. Otowa K, Takamura M, Murai H, Maruyama M, Nakano M, Ikeda T, Kobayashi D, Ootsuji H, Okajima M, Furushou H, Yuasa T, Takata S, Kaneko $\mathrm{S}$. Altered interaction between lasminogen activator inhibitor type 1 activity and sympathetic nerve activity with aging. Circ J 2008;72:458-62.

29. Bove EL, Fry WJ, Gross WS, Stanley JC. Hypotension and hypertension as consequences of baroreceptor dysfunction following carotid endarterectomy. Surgery 1979;85:633-7.

30. Hirschl M, Kundi M, Blazek G. Five-year follow-up of patients after thromboendarterectomy of the internal carotid artery: Relevance of baroreceptor sensitivity. Stroke 1996;27:1167-72. 